\title{
Corrigendum to "Formation of solid particles in synoptic-scale Arctic PSCs in early winter 2002/2003" published in Atmos. Chem. Phys., 4, 2001-2013, 2004
}

\section{N. Larsen et al.}

Danish Meteorological Institute, Lyngbyvej 100, DK-2100 Copenhagen, Denmark

The correct acknowledgement reads as follows:

We would like to thank the team from Centre National d'Etude Spatiales, especially the late P. Faucon, for excellent handling of the balloon-flights, and people from the Swedish Space Corporation at Esrange for their efficient support to the experiments. We would like to extend our thanks to the European Space Agency for early provision of MIPAS-Envisat $\mathrm{HNO}_{3}$ profiles. The T-matrix code has been obtained from M. I. Mishchenko's web site. B. P. Luo and C. Voight are ac- knowledged for their contributions in establishing a T-matrix expansion coefficient data base. This work was supported by the EU Commission projects CIPA (contract EVK2-CT2000-00095) and MAPSCORE (contract EVK2-CT-200000072), the German Bundesministerium für Bildung und Forschung (BMBF, AFO-2000) POSTA project, the US National Science Foundation (contracts OPP- 0095158 and ATM-0086484), and the Danish National Science Foundation. 